\title{
Internet Use and Anxiety in People with Melanoma and Nonmelanoma Skin Cancer
}

\author{
Mathew W. Ludgate, MBChB, ${ }^{*}$ Michael S. Sabel, MD, ${ }^{\dagger}$ Douglas R. Fullen, MD,$* \ddagger$ \\ Marcus L. Frohm, MD, ${ }^{*}$ Julia S. Lee, MS,${ }^{\S}$ Mick P. Couper, PhD, ${ }^{\S}$ Timothy M. Johnson, MD, ${ }^{* \dagger}$ \\ AND Christopher K. BichakJian, MD*
}

BACKGROUND People with cancer are increasingly turning to the Internet for health-related information.

OBJECTIVE To compare the patterns of Internet use of people with skin cancer with previous findings by including people with nonmelanoma skin cancer (NMSC) using a comprehensive survey. To evaluate perceived anxiety levels and overall satisfaction after searching the Internet of people with skin cancer.

METHODS \& MATERIALS We conducted a survey study and prospectively collected data from people newly diagnosed with melanoma or NMSC.

RESULTS Four hundred fifteen participants with melanoma and 400 with NMSC completed the questionnaire. Internet use and overall satisfaction with the Internet search increased more than $50 \%$ in participants with melanoma from 2005. One-third of participants with melanoma, but many fewer participants with NMSC, reported higher anxiety after Internet use. Participants who were younger, female, more highly educated, and diagnosed with melanoma were most likely to use the Internet to search for information about their diagnosis.

CONCLUSION Internet use is prevalent and increasing sharply in individuals with skin cancer. The majority of individuals describe their use of the Internet as a positive experience. Greater anxiety from searching the Internet is more common in individuals with melanoma than in those with NMSC.

The authors have indicated no significant interest with commercial supporters.

$\mathrm{O}$ ver the past decade, the Internet has emerged as an increasingly popular source of healthrelated information. Internet use has grown at an astonishing rate, and it is estimated that at least $74 \%$ of the U.S. population currently uses the Internet; ${ }^{1}$ $80 \%$ of U.S. Internet users, amounting to approximately 113 million adults, have reported searching for at least one major health topic online. ${ }^{2}$

Although the diagnosis of cancer is unwelcome news to individuals and their families, the majority of people with cancer who have been surveyed wish to have as much information as possible pertaining to their disease. ${ }^{3-5}$ Physician consultations typically serve as the primary source of this information. Although most individuals rate the information that their physician provides as the most important source, ${ }^{6-8}$ a significant proportion are dissatisfied with the information provided. ${ }^{8,9}$ As such, many people with cancer are turning to the Internet for additional information, with an estimated $39 \%$ of people with cancer reporting use of the Internet and an additional $15 \%$ to $20 \%$ reporting indirect Internet use through family and friends. ${ }^{10}$ For many individuals diagnosed with skin cancer, the Internet may serve as a valuable resource, but the overwhelming volume of information, as well as potential conflicting or inaccurate information, may also result in confusion and anxiety. Although most melanoma websites provide extensive and valuable information, as many as $14 \%$ may contain inaccuracies. ${ }^{11}$ An earlier study performed several years ago, asking three short questions, showed that Internet use in people with melanoma is common with

Departments of ${ }^{*}$ Dermatology, ${ }^{\dagger}$ Surgery, ${ }^{\ddagger}$ Pathology, and ${ }^{\top}$ Otolaryngology, University of Michigan Health System and ${ }^{\S}$ Institute for Social Research, University of Michigan, Ann Arbor, Michigan 
a variable effect on anxiety. Approximately one-third of people with melanoma reported greater anxiety as a result of their melanoma-related Internet use. ${ }^{12}$

We designed the current study to evaluate patterns of Internet use in people newly diagnosed with skin cancer with the intent of validating and expanding on previous findings with a far more comprehensive survey. Although the current study involved fewer participants than our previous study, a more indepth and balanced questionnaire was designed, allowing participants to elaborate on questions in more detail and allowing more-robust validation of our previous findings. We broadened our participant population to include participants with basal cell carcinoma (BCC) or squamous cell carcinoma (SCC) to assess whether the diagnosis of skin cancer in general leads to similar patterns of health information-seeking behavior regardless of prognosis. To determine the effect the use of the Internet had on people with skin cancer, we evaluated participants' perceived anxiety levels and overall satisfaction after they had searched the Internet.

\section{Materials and Methods}

Study approval was obtained from the University of Michigan Institutional Review Board for Human Subject Research. Data collection was conducted between January 2006 and May 2007 and consisted of three random sampling periods each lasting between 2 and 6 consecutive months. During these periods, all individuals scheduled for an initial consultation in the Multidisciplinary Melanoma Clinic at the University of Michigan with a presumed diagnosis of melanoma were contacted by telephone before their appointment. Individuals were asked about their willingness to arrive 15 to 30 minutes before their appointment to complete a survey questionnaire. The topic of the survey was purposely kept vague to avoid the ability to prepare for the questionnaire. During the same time periods, the same procedure was followed for individuals with a presumed diagnosis of BCC or SCC scheduled for an initial consultation in the Cutaneous Surgery and
Oncology Unit at the University of Michigan. Individuals younger than 18 and those who had previously been seen in the Multidisciplinary Melanoma Clinic were excluded, and individuals with multiple primary skin cancers were only included once, with data obtained from the first consultation. The number of individuals unwilling or unable to participate and the reasons for nonparticipation were recorded. All participants consented to the inclusion of demographic and clinical data in our prospective melanoma database.

Eligible individuals completed a survey questionnaire upon their arrival, before the face-to-face physician encounter. The survey was originally piloted as a brief questionnaire consisting of three questions providing preliminary data, which was previously published. ${ }^{12}$ The final survey instrument used in this study was designed in close collaboration with a leading survey methodologist (MC) in survey research at the University of Michigan. The questionnaire consisted of 24 , mostly multiple-choice questions related to skin cancer, Internet use, and demographic information (Appendix 1). Anxiety was assessed by multiple-choice, using a 5-point scale ranging from much less anxious to much more anxious. At the beginning of each consultation, the attending physician asked participants about their knowledge of their disease with regard to diagnosis, Breslow depth, extent (localized, regional, or distant), and location (face, head and neck nonface, trunk, or extremity), as applicable to their malignancy, and the responses were recorded.

To confirm the diagnosis of melanoma in study participants, a board-certified dermatopathologist reviewed all histologic slides. Clinical information was extracted from our prospectively collected melanoma database, including age at diagnosis; sex; Breslow depth; and presence or absence of in-transit, regional lymphatic or distant metastases at the time of initial presentation. Similar to our previous pilot study, primary melanomas were classified as melanoma in situ, Breslow depth less than $1 \mathrm{~mm}$, Breslow depth 1 to $4 \mathrm{~mm}$, and Breslow depth greater 
than $4 \mathrm{~mm}$. Participants with melanomas of unknown Breslow depth were excluded from analyses involving depth but were otherwise included.

Descriptive statistics such as means and proportions are reported. The chi-square test was used to assess associations between categorical values, and the Wilcoxon rank sum test was used to assess the association between a continuous variable (such as age at diagnosis) and a two-level categorical variable. $P<.05$ was considered to be statistically significant, and all of the tests were two-sided.

\section{Results}

\section{Study Population}

Four hundred fifteen participants with melanoma and 400 with nonmelanoma skin cancer (NMSC) completed the survey questionnaire during the study period. Twenty-two individuals were previously seen in our melanoma clinic and were therefore excluded. Demographic characteristics of the remaining
793 participants are listed in Table 1. An additional 202 individuals were contacted but were unable or unwilling to complete the survey for reasons summarized in Table 2.

The majority of 265 participants with invasive melanoma had clinically localized disease at the time of consultation. Only five presented with nodal metastases (stage III) and four with distant disease (stage IV). The mean Breslow depth of melanomas was $1.27 \mathrm{~mm}$ (range $0.15-10 \mathrm{~mm}$ ). The reasons for unknown Breslow depth in 11 participants included a diagnosis of invasive melanoma without depth specified by outside pathologist not confirmed as melanoma by our dermatopathologist, mucosal (conjunctival/oral) melanoma without specified Breslow depth, unknown primary melanoma, and newly diagnosed stage III disease and unavailability of records from original primary melanoma.

Before receiving a diagnosis of skin cancer, $90 \%$ of participants with melanoma had previously heard of

\section{TABLE 1. Patient Characteristics}

\begin{tabular}{|c|c|c|c|}
\hline Characteristic & Melanoma & Nonmelanoma Skin Cancer & P-Value \\
\hline Age, mean & 56 & 66 & $<.001^{\dagger}$ \\
\hline Sex, \% & & & $.6^{*}$ \\
\hline Male & 46 & 44 & \\
\hline Female & 54 & 56 & \\
\hline First skin cancer, \% & 72 & 42 & $<.001^{*}$ \\
\hline \multicolumn{4}{|l|}{ Tumor, \% } \\
\hline Melanoma & 395 & & \\
\hline In situ & 130 & & \\
\hline$<1 \mathrm{~mm}$ & 140 & & \\
\hline $1-4 \mathrm{~mm}$ & 93 & & \\
\hline$>4 \mathrm{~mm}$ & 21 & & \\
\hline Unknown depth & 11 & & \\
\hline NMSC & & 398 & \\
\hline Squamous cell carcinoma & & 108 & \\
\hline Basal cell carcinoma & & 290 & \\
\hline Education level, \% & & & $.08^{*}$ \\
\hline Postgraduate or professional degree & 23 & 21 & \\
\hline College degree & 21 & 25 & \\
\hline Associate degree or technical school & 14 & 14 & \\
\hline High school or general educational development & 36 & 37 & \\
\hline Less than high school & 7 & 3 & \\
\hline
\end{tabular}


TABLE 2. Reasons for Inability or Unwillingness to Complete Study Questionnaire

\begin{tabular}{lll} 
& $\mathrm{n}(\%)$ & \\
\cline { 2 - 3 } Reason & Melanoma & Nonmelanoma Skin Cancer \\
\hline Late arrival & $49(35)$ & $26(41)$ \\
Not contactable before appointment & $54(39)$ & $13(21)$ \\
Physically or mentally unable to complete survey & $8(6)$ & $9(9)$ \\
Age $<18$ & $6(4)$ & 0 \\
No definitive diagnosis at time of consultation & 0 & $2(3)$ \\
Declined & $22(16)$ & $13(21)$ \\
Total & $139(100)$ & $63(100)$ \\
\hline
\end{tabular}

melanoma, as had $89 \%$ of participants with NMSC. Participants with NMSC were significantly more likely to have heard of BCC and SCC $(75 \%$ and $56 \%$, respectively) than those with melanoma $(66 \%$ and $48 \%, p<.05)$. The majority of both groups of participants read or watched health information at least once a month $(74 \%)$. Women reported that they were more likely to read or watch health information at least weekly than men ( $46 \%$ vs $28 \%$, $p<.001)$ and indicated that they paid more attention to their health than men ( $65 \%$ vs $53 \%, p=.003)$. There was no significant difference in mean age between participants who read or watched health information frequently and those who did not $(p=.10)$, although older participants reported paying greater attention to their health than younger participants $(p=.001)$.

\section{Internet Use}

Seventy-four percent of participants with melanoma and $61 \%$ with NMSC had accessed the Internet or used email in the 12 months before their diagnosis. Participants without Internet access were significantly older than those with access (Wilcoxon rank sum test, $p<.001)$. Participants with Internet access were significantly more likely than those without to have heard of BCC $(74 \%$ vs $63 \%, p<.05)$ and melanoma $(93 \%$ vs $84 \%, p<.05)$ before their diagnosis. Participants with Internet access were also more likely to read or watch health information at least once weekly than those without $(41 \%$ vs $27 \%$, $p<.05)$.

\section{Patterns of Internet Use}

Regardless of their type of skin cancer, the majority of participants were satisfied with the amount of information provided by their referring physician (95\% of participants with melanoma and $91 \%$ with NMSC). Despite this, a large number of participants sought additional information regarding their diagnosis on the Internet, as summarized in Table 3. Participants with skin cancer who searched the Internet themselves for information related to their diagnosis were more likely to be younger (mean age 52.5 vs $64.4, p<.001$ ), to be female ( $55 \%$ vs $46 \%$ of men, $p<.001$ ), to have been diagnosed with melanoma ( $48 \%$ vs $23 \%$ of NMSC, $p<.001)$, and to be more-highly educated $(60 \%$ professional or college level vs $27 \%<$ high school, $p<.001)$.

Participants who used the Internet were generally highly satisfied and positive about their Internet experience, with $90 \%$ of participants reporting that they would recommend the Internet to others, and $75 \%$ reporting greater understanding of their diagnosis and disease management. Although a significant number of participants with melanoma reported that the Internet raised questions about their condition or that they found information that they had not previously received from a physician, only $41 \%$ of these reported that they planned to discuss this with their physician. Women were more likely than men to use the Internet to verify the information they received from their physician $(28 \%$ vs $17 \%, p<.05)$. More than half of all study participants (59\%) reported that they trusted only some of the 
TABLE 3. Comparison of Internet Use Characteristics of Patients with Melanoma and Nonmelanoma Skin Cancer (NMSC)

\begin{tabular}{|c|c|c|c|}
\hline \multirow[b]{2}{*}{ Internet Use } & \multicolumn{2}{|l|}{$\%$} & \multirow[b]{2}{*}{ P-Value* } \\
\hline & Melanoma & NMSC & \\
\hline \multicolumn{4}{|l|}{ Internet Use } \\
\hline Perform search themselves & 48 & 23 & $<.001$ \\
\hline Perform search via family/friends & 35 & 17 & $<.001$ \\
\hline \multicolumn{4}{|l|}{ Source of website } \\
\hline Search engine & 69 & 49 & .002 \\
\hline Physician & 11 & 4 & .008 \\
\hline Media & 2 & 2 & .90 \\
\hline Friend or family & 7 & 9 & .67 \\
\hline Health website & 20 & 18 & .60 \\
\hline \multicolumn{4}{|l|}{ Website searched } \\
\hline American Cancer Society (www.cancer.org) & 42 & 24 & .001 \\
\hline National Cancer Institute (www.nci.nih.gov) & 28 & 7 & $<.001$ \\
\hline Skin Cancer Foundation (www.skincancer.org) & 23 & 21 & .84 \\
\hline American Academy of Dermatology (www.aad.org) & 14 & 8 & .26 \\
\hline Complementary medicine sites & 11 & 5 & .09 \\
\hline Unknown & 42 & 24 & .0005 \\
\hline \multicolumn{4}{|l|}{ Reason for searching Internet } \\
\hline Find information about condition & 91 & 68 & $<.001$ \\
\hline Verify information from doctor & 24 & 20 & .34 \\
\hline Search for complementary approaches & 17 & 12 & .12 \\
\hline Search for clinical trials & 9 & 4 & .04 \\
\hline \multicolumn{4}{|l|}{ Usefulness of Internet } \\
\hline Helped with understanding of condition & 78 & 73 & .31 \\
\hline Found new information not provided by physician & 51 & 28 & $<.001$ \\
\hline Raised questions about diagnosis or management & 41 & 17 & $<.001$ \\
\hline
\end{tabular}

information they found on the Internet, whereas $30 \%$ trusted all of the information found. Participants with more deeply invasive melanomas were more likely to search for information on clinical trials than participants with thinner melanomas $(p<.05)$.

Participants with skin cancer searching complementary medicine websites were more likely to have melanoma, to be younger, to be female, and to have a higher level of education than those who did not seek out these specific sites, although this did not reach statistical significance. Participants with deeper melanomas were not more likely to search complementary medicine websites.

\section{Anxiety}

Of participants with melanoma who had used the Internet, approximately one-third reported that their search had made them more anxious, one-third felt less anxious, and one-third felt neutral (Table 4). In participants with NMSC, greater anxiety was

TABLE 4. Anxiety Levels of Participants with Melanoma and Nonmelanoma Skin Cancer (NMSC) After Use of the Internet as a Source of Medical Information with Regard to Diagnosis

\begin{tabular}{lll} 
& \multicolumn{2}{l}{$\%$} \\
\cline { 2 - 3 } Anxiety Level & Melanoma* $^{N}$ & NSS $^{\dagger}$ \\
\hline Much less & 6 & 16 \\
Slightly less & 24 & 23 \\
Neutral & 35 & 48 \\
Slightly more & 25 & $10^{\ddagger}$ \\
Much more & 11 & $3^{\ddagger}$ \\
\hline
\end{tabular}

*Percentage of participants with melanoma who used the Internet. 'Percentage of participants with NMSC who used the Internet. ${ }^{\ddagger} p<.001$; chi-square test. 
significantly less common. Participants who indicated that the use of the Internet had increased their level of anxiety were specifically asked why it had made them more anxious. Among the most common answers were greater awareness of the potential seriousness of their diagnosis, confusion and uncertainty, unanswered questions about their specific condition, being confronted with the worst-case scenario, mention of survival rates, and too much information in general. One participant summarized it as "reality." When participants who indicated that their Internet search had made them less anxious were asked to explain, the most common answer was a better understanding of their disease. A participant who indicated that "information is the power to conquer the fear of the unknown" best summarized the sentiment in this group.

An inverse correlation was found between age and increasing anxiety level, with younger participants significantly more anxious than older participants (Spearman correlation $p=.006$ ). There was a trend toward participants with deeper melanomas being more likely to report greater anxiety from Internet use than participants with melanoma in situ. No correlation was found between level of anxiety and sex, education level, level of prior interest in health, or prior frequency of reading or watching health information.

\section{Discussion}

In this study, we found that the number of participants diagnosed with melanoma who used the Internet to search for information regarding their diagnosis had risen sharply, from $39 \%$ to $83 \%$, compared with our previous analysis in 2005, combining participants who searched the Internet themselves and those whose family or friends searched for them ${ }^{12}$ (Figure 1 ). Although this was significantly lower in participants diagnosed with BCC or SCC, the percentage was still nearly $40 \%$. A substantial increase in individuals reporting that they would recommend the Internet to others, from $45 \%$ in 2005 to $90 \%$ in this study, is evidence of the high

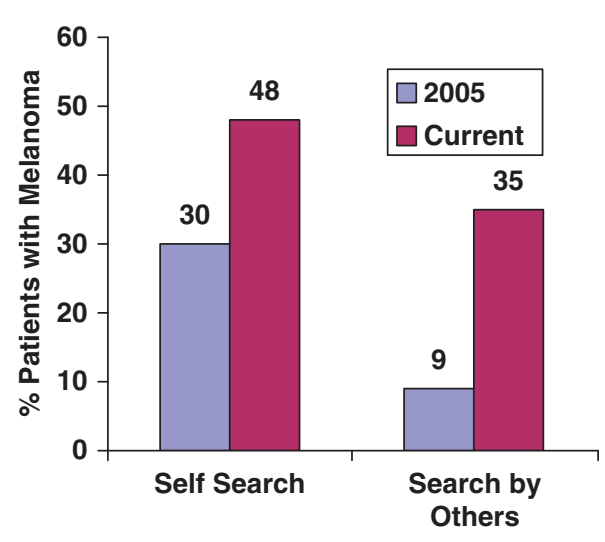

Figure 1. Percentage of participants with melanoma searching the Internet or asking others to search for medical information regarding their diagnosis in current study versus 2005. ${ }^{12}$

level of satisfaction with use of the Internet as an information source. ${ }^{12}$ This is similar to previous studies in oncology populations that reported a high level of satisfaction with Internet use. ${ }^{7,8}$ These findings highlight the increasing importance of the Internet as a provider of information on skin cancer and health-related concerns in general.

Although people are enthusiastic about Internet use, physicians often express concern about individuals' ability to evaluate the vast amount of information and place it in the proper context as it relates to their diagnosis. Moreover, previous studies have shown that some health-related websites contain incomplete, out-of-date, inaccurate information. ${ }^{11,13,14}$ Despite this potential for harm, the majority of participants in our study did not report greater anxiety from their Internet use, and for most people, the Internet served as a valuable resource. This was particularly true for participants with BCC and SCC, of whom only $13 \%$ reported any increase in anxiety after their Internet search regarding their diagnosis. In participants with melanoma, we confirmed, with a far-more-comprehensive survey, our previous findings that approximately one-third of individuals experience an increase in anxiety regarding their diagnosis as a consequence of their Internet search. ${ }^{12}$ In contrast with our prior study, the depth of the primary melanoma was not significantly correlated with anxiety, although a trend existed. ${ }^{12}$ 
This difference may also be the result of a smaller sample size.

As expressed by our participants, Internet-related anxiety was attributed to many factors, such as heightened awareness of the potential severity of the diagnosis, difficulty understanding mortality data, confusion with staging, feelings of hopelessness in participants with advanced melanoma, and fear of potential therapies. In our experience, the staging classifications of their malignancy confuse many individuals recently diagnosed with melanoma. Specifically, the histopathologic classification of Clark level (e.g., level IV) is often mistaken for the clinical American Joint Committee on Cancer (AJCC) staging system (e.g., stage IV), which is also given in Roman numerals. ${ }^{15}$ This frequently leads people to believe that their disease is far more advanced than it really is, resulting in significant yet unfounded anxiety. Health care providers should be mindful that rather technical medical information, such as a pathology report, however well intended, may easily be misinterpreted without a thorough explanation. In this specific example, an up-front discussion of the AJCC staging system for people with melanoma may prevent individuals from staging their own disease online and avoid unnecessary distress. Conversely, some people who report less anxiety resulting from their Internet use, may be overly optimistic about their prognosis or treatment options. ${ }^{16}$ This could, in turn, result in delayed anxiety when the individual is faced with a more-balanced view of his or her disease and prognosis.

Nearly two-thirds of participants reported that they trusted only some of the information they found on the Internet, suggesting that most people have an appropriate degree of caution with the Internet. Although people may display caution regarding the trustworthiness of the Internet, a low percentage reported that they would discuss any questions raised by the Internet with their physician $(41 \%)$. This suggests that many people who use the Internet and become confused may remain so, which could result in greater anxiety and distress. To minimize this possibility, it appears prudent for health care providers to be proactive in questioning whether people have used the Internet and asking whether this search has raised any questions or concerns.

In contrast with previous studies, ${ }^{8,9}$ we found that the majority of participants were satisfied with the amount of information they had received from their referring physician about their diagnosis. A potential reporting bias could exist because study participants may see researchers at our institution as connected with their providers. Despite the high levels of satisfaction, a significant proportion of participants searched the Internet for further information on their disease, suggesting that people may use the Internet to find different information than what they expect to receive from health care providers. Alternatively, searching for information may be a coping strategy that some people use who are satisfied with the information they received but wish to reassure themselves that they have all the information available. ${ }^{17}$

Similar to previous studies, we found that Internet search engines were the most common source of websites. ${ }^{18}$ The most frequently visited websites are listed in Table 3. Websites from major national institutions, most notably the American Cancer Society (http://www.cancer.org), were among the most frequently mentioned sites, although approximately one-third of participants did not recall which websites they had visited. Participants with melanoma used websites recommended by a health care provider significantly more often than participants with NMSC. This may be attributed to the fact that referring physicians are more likely to recommend websites with a diagnosis of melanoma. Nonetheless, these findings highlight that people are responsive to health care provider website recommendations, which may provide a valuable means of influencing people's Internet use.

In conclusion, Internet use as a source of medical information has risen sharply in people diagnosed with skin cancer. People with skin cancer who were younger, female, more highly educated, and 
diagnosed with melanoma were most likely to use the Internet to search for information about their diagnosis. The majority of participants described their use of the Internet as a positive experience, which they would recommend to others, yet viewed the content of the available websites with appropriate caution. Although Internet use does not lead to greater anxiety in most people, more than one-third of people diagnosed with melanoma experienced higher levels of anxiety as a result of their Internet search. Greater anxiety from Internet use is less common in people with NMSC, although given the increasing incidence of BCC and SCC in younger, more-Internet-savvy people, health care providers should be aware that people with NMSC may experience similar Internet-related anxiety, despite a favorable prognosis. ${ }^{19,20}$ A proactive role by health care providers could maximize the benefits of Internet use by recommending suitable Websites and identifying people who have used the Internet and addressing their concerns.

\section{Acknowledgments This study was funded by a} Dermatology Foundation Patient Directed Investigation Grant (\#N007694), Principal Investigator C.K.B.

\section{References}

1. Internet Usage Statistics for the Americas. Available at:http:// Internetworldstats.com/stats2.htm. Accessed January 17, 2010.

2. Fox S. Online Health Search 2006. Available at:http://www. pewInternet.org. Accessed January 31, 2010.

3. Hay J, Coups EJ, Ford J, DiBonaventura M. Exposure to mass media health information, skin cancer beliefs, and sun protection behaviors in a United States probability sample. J Am Acad Dermatol 2009;61:783-92.

4. Meredith C, Symonds P, Webster L, Lamont D, et al. Information needs of cancer patients in west Scotland: cross sectional survey of patients' views. BMJ 1996;313:724-6.

5. Jenkins V, Fallowfield L, Saul J. Information needs of patients with cancer: results from a large study in UK cancer centres. Br J Cancer 2001;84:48-51.

6. Diefenbach MA, Dorsey J, Uzzo RG, Hanks GE, et al. Decisionmaking strategies for patients with localized prostate cancer. Semin Urol Oncol 2002;20:55-62.
7. Peterson MW, Fretz PC. Patient use of the Internet for information in a lung cancer clinic. Chest 2003;123:452-7.

8. Chen X, Siu LL. Impact of the media and the Internet on oncology: survey of cancer patients and oncologists in Canada. J Clin Oncol 2001;19:4291-7.

9. Jones R, Pearson J, McGregor S, Gilmour WH, et al. Cross sectional survey of patients' satisfaction with information about cancer. BMJ 1999;319:1247-8.

10. Eysenbach G. The impact of the Internet on cancer outcomes. CA Cancer J Clin 2003;53:356-71.

11. Bichakjian CK, Schwartz JL, Wang TS, Hall JM, et al. Melanoma information on the Internet: often incomplete-a public health opportunity? J Clin Oncol 2002;20:134-41.

12. Sabel MS, Strecher VJ, Schwartz JL, Wang TS, et al. Patterns of Internet use and impact on patients with melanoma. J Am Acad Dermatol 2005;52:779-85.

13. Black PC, Penson DF. Prostate cancer on the Internet-information or misinformation? J Urol 2006;175:1836-42; discussion 1842.

14. Eysenbach G, Powell J, Kuss O, Sa ER. Empirical studies assessing the quality of health information for consumers on the world wide web: a systematic review. JAMA 2002;287:2691-700.

15. Balch CM, Gershenwald JE, Soong SJ, Thompson JF, et al. Final version of 2009 AJCC melanoma staging and classification. J Clin Oncol 2009;27:6199-206.

16. Helft PR, Hlubocky F, Daugherty CK. American oncologists' views of Internet use by cancer patients: a mail survey of American Society of Clinical Oncology members. J Clin Oncol 2003;21:942-7.

17. Derdiarian AK. Informational needs of recently diagnosed cancer patients. Nurs Res 1986;35:276-81.

18. Eysenbach G, Kohler C. How do consumers search for and appraise health information on the world wide web? Qualitative study using focus groups, usability tests, and in-depth interviews. BMJ 2002;324:573-7.

19. Bath-Hextall F, Leonardi-Bee J, Smith C, Meal A, et al. Trends in incidence of skin basal cell carcinoma. Additional evidence from a UK primary care database study. Int J Cancer 2007; 121:2105-8.

20. Rogers HW, Weinstock MA, Harris AR, Hinckley MR, et al. Incidence estimate of nonmelanoma skin cancer in the United States, 2006. Arch Dermatol 2010;146:283-7.

Address correspondence and reprint requests to: Christopher K. Bichakjian, MD, University of Michigan Health System, 1910 Taubman Center, 1500 East Medical Center Drive SPC 5314, Ann Arbor, MI 48109-5314, or e-mail: chriskb@med.umich.edu 